PROCEEDINGS OF THE

AMERICAN MATHEMATICAL SOCIETY

Volume 132, Number 4, Pages 1103-1111

S 0002-9939(03)07150-8

Article electronically published on September 18, 2003

\title{
MINIMAL DISPLACEMENT AND RETRACTION PROBLEMS IN INFINITE-DIMENSIONAL HILBERT SPACES
}

\author{
KRZYSZTOF BOLIBOK
}

(Communicated by N. Tomczak-Jaegermann)

\begin{abstract}
We give the first constructive example of a Lipschitz mapping with positive minimal displacement in an infinite-dimensional Hilbert space $H$. We use this construction to obtain an evaluation from below of the minimal displacement characteristic in the space $H$. In the second part we present a simple and constructive proof of existence of a Lipschitz retraction from a unit ball $B$ onto a unit sphere $S$ in the space $H$, and we improve an evaluation from above of a retraction constant $k_{0}(H)$.
\end{abstract}

\section{INTRODUCTION}

Let $(X,\|\cdot\|)$ be an infinite-dimensional Banach space with the closed unit ball $B$ and the unit sphere $S$. For any $k \geq 0$, let $L(k)$ denote the class of Lipschitz mappings with constant $k$. If $K$ is a bounded, closed and convex subset of $X$, then by $d_{T}$ we will denote minimal displacement of $T$,

$$
d_{T}=\inf _{x \in K}\|x-T x\|,
$$

where $T: K \rightarrow K$. This notion for Lipschitz mappings was introduced by Goebel [7] in 1973. In this paper he gave examples of sets $K$ and Lipschitz mappings $T$ with positive $d_{T}$. The problem for which sets there exists a Lipschitz mapping with positive minimal displacement was studied by many authors, and a final solution was given by Lin and Sternfeld [12. They proved that if $K$ is bounded, closed and convex but noncompact, then there exists a Lipschitz mapping $T$ such that $d_{T}>0$. On the other hand, very little is known about qualitative properties of minimal displacement. Goebel in the above-mentioned paper introduced some functions describing the minimal displacement problem. We recall two of them. The first, denoted by $\varphi_{X}$, is defined as follows:

$$
\varphi_{X}(k)=\sup \left\{d_{T}: K \subset X, r(K)=1, T: K \rightarrow K, T \in L(k), k \geq 1\right\},
$$

where $r(K)$ is the Chebyschev radius of $K$, i.e.,

$$
r(K)=\inf [\sup \{\|x-y\|: x \in K\}: y \in X] .
$$

Received by the editors November 6, 2001 and, in revised form, December 10, 2002.

2000 Mathematics Subject Classification. Primary 47H09, 47H10.

Key words and phrases. Lipschitz mappings, minimal displacement, Lipschitz retraction.

This research was supported in part by KBN grant 2 PO3A 02915. 
The second $\psi_{X}$ denotes the minimal displacement characteristic of $X$,

$$
\psi_{X}(k)=\sup \left\{d_{T}: T: B \rightarrow B, T \in L(k), k \geq 1\right\} .
$$

It is known that for any space $X$,

$$
\psi_{X}(k) \leq \varphi_{X}(k) \leq 1-\frac{1}{k} .
$$

Moreover, the following holds:

$$
\begin{aligned}
\psi_{X}(1-\alpha+\alpha k) & \geq \alpha \psi_{X}(k) \text { for any } \alpha \in[0,1], \\
\lim _{k \rightarrow \infty} \psi_{X}(k) & =1 \text { for any space } X .
\end{aligned}
$$

There are some "square" spaces like $c_{0}, C[0,1]$ for which $\psi_{X}(k)=\varphi_{X}(k)=1-\frac{1}{k}$. The two functions are equal in the Hilbert space $H$ and the following estimate holds:

$$
\psi_{H}(k)=\varphi_{H}(k) \leq\left(1-\frac{1}{k}\right) \sqrt{\frac{k}{k+1}} .
$$

In 1979 Nowak [13] proved that in some infinite-dimensional Banach spaces there exist Lipschitz retractions of unit balls onto unit spheres. Four years later Benyamini and Sternfeld [1] extended the above result to all infinite-dimensional Banach spaces. This result leads directly to the definition of the so-called retraction constant $k_{0}(X)$, being the infimum of the set of all numbers $k>1$ for which there exists a retraction $R: B \rightarrow S$ belonging to $L(k)$. It known that $k_{0}(X) \geq 3$ for any space $X$. For the space $H$ we know that $k_{0}(H)>4.5$. On the other hand, Komorowski and Wośko [11] gave an example of a Lipschitz retraction with Lipschitz constant less than 64.5. Unfortunately their example is not completely constructive. For a wider discussion of the topics mentioned above we refer the reader to [3]- $[6]$, 9]-11], [14, and [15].

\section{Minimal Displacement}

For more than twenty years since Goebel's paper there have been no results on the minimal displacement problem in the space $H$. In 1996 the author 2] proved the following evaluation from below of the function $\psi_{H}$.

Theorem 2.1. In an infinite-dimensional Hilbert space $H$,

$$
\psi_{H}(k) \geq \sup _{\epsilon \in(0,2)}\left(1-\frac{2+\epsilon}{\sqrt{1+\epsilon(\epsilon+2) k^{2}}-1}-\epsilon(k+1)\right) .
$$

Unfortunately this evaluation is very imprecise. Below we present a better estimation. Let us consider the Hilbert space $L^{2}(0,1)$ and let $S^{+}$denote the nonnegative part of the unit sphere $S$, i.e.,

$$
S^{+}=\{x \in S: x(t) \geq 0 \text { for } t \in(0,1)\} .
$$

By $d_{T}$ denote minimal displacement also in this setting $\left(S^{+}\right.$is not convex). We start with the following lemma.

Lemma 2.2. For every $\alpha \geq 2$ there exists a mapping $T: S^{+} \rightarrow S^{+}$from the class $L\left(\alpha^{\frac{3}{2}}\right)$ such that $d_{T}=\frac{\sqrt{2}(\sqrt{\alpha}-1)}{\sqrt{\alpha+1}}$. 
Proof. Let $\alpha \geq 2$ and let the mapping $T: S^{+} \rightarrow S^{+}$be defined by an equality

$$
\int_{0}^{t}[(T x)(s)]^{2} d s=\left(\int_{0}^{t}[x(s)]^{2} d s\right)^{\alpha} \quad \text { for every } t \in(0,1) .
$$

Observe that the map is well defined and that it can be written equivalently as

$$
(T x)(t)=\sqrt{\alpha} x(t)\|x\|_{t}^{\alpha-1},
$$

where

$$
\|x\|_{t}=\sqrt{\int_{0}^{t}[x(s)]^{2} d s .}
$$

First we show that $T$ is a Lipschitz mapping.

$$
\begin{aligned}
\|T x-T y\|^{2} & =\alpha \int_{0}^{1}\left[x(t)\|x\|_{t}^{\alpha-1}-y(t)\|y\|_{t}^{\alpha-1}\right]^{2} d t \\
& \leq \alpha \int_{0}^{1}\left[x(t)\left|\|x\|_{t}^{\alpha-1}-\|y\|_{t}^{\alpha-1}\right|+|x(t)-y(t)|\|y\|_{t}^{\alpha-1}\right]^{2} d t \\
& \leq \alpha \int_{0}^{1}\left[(\alpha-1) x(t)\left|\|x\|_{t}-\|y\|_{t}\right|+|x(t)-y(t)|\right]^{2} d t \\
& \leq \alpha \int_{0}^{1}[(\alpha-1) x(t)\|x-y\|+|x(t)-y(t)|]^{2} d t .
\end{aligned}
$$

Furthermore using standard arguments such as the triangle and Cauchy-Schwartz inequalities, we get

$$
\|T x-T y\|^{2} \leq \alpha^{3}\|x-y\|^{2} .
$$

Hence $T \in L\left(\alpha^{\frac{3}{2}}\right)$. The minimal displacement of $T$ can be written as follows:

$$
\begin{aligned}
\|x-T x\|^{2} & =\|x\|^{2}-2\langle x, T x\rangle+\|T x\|^{2} \\
& =2-2 \int_{0}^{1} x(t)(T x)(t) d t \\
& =2-2 \sqrt{\alpha} \int_{0}^{1}[x(t)]^{2}\left(\int_{0}^{t}[x(s)]^{2} d s\right)^{\frac{\alpha-1}{2}} d t .
\end{aligned}
$$

Setting

$$
z=\int_{0}^{t}[x(s)]^{2} d s
$$

we get

$$
\|x-T x\|^{2}=2-2 \sqrt{\alpha} \int_{0}^{1} z^{\frac{\alpha-1}{2}} d z=\frac{2(\sqrt{\alpha}-1)^{2}}{\alpha+1}>0 .
$$

Observe that $\|x-T x\|=$ const for every $x \in S^{+}$. Moreover, when $\alpha \rightarrow \infty$, then $\|x-T x\| \rightarrow \sqrt{2}=\operatorname{diam} S^{+}$.

Theorem 2.3. Let $H$ be an infinite-dimensional Hilbert space. Then

$$
\psi_{H}\left(\sqrt{2}(\alpha+1) \alpha^{\frac{3}{2}}\right) \geq \frac{\alpha-1}{\alpha+1} \quad \text { for } \alpha \geq 2 .
$$


Proof. For any $r>0$, let $B^{+}(r)$ be the nonnegative part of the ball $B(r)$ of radius $r$, i.e., the set

$$
B^{+}(r)=\{x \in B(r): x(t) \geq 0 \text { for } t \in(0,1)\} .
$$

Define a mapping $T_{1}: B \rightarrow B^{+}(1)$ by

$$
\left(T_{1} x\right)(t)=|x(t)|
$$

Obviously, $T_{1} \in L(1)$. Let $\delta$ denote the following expression:

$$
\delta=d_{T} \sqrt{1-\left(\frac{d_{T}}{2}\right)^{2}}=\frac{\alpha-1}{\alpha+1},
$$

where $d_{T}$ is the minimal displacement of the map $T$ from the previous lemma. Furthermore, we define a map $\widetilde{T}$ such that $d_{\widetilde{T}}=\delta$. But now let a mapping $T_{2}$ : $B^{+}(1) \rightarrow B^{+}(1)$ be given as

$$
\left(T_{2} x\right)(t)= \begin{cases}x(t) & \text { for }\|x\| \geq 1-\delta \\ 1-\delta-\|x\|+x(t) & \text { for }\|x\|<1-\delta\end{cases}
$$

Obviously when $\|x\| \geq 1-\delta$ and $\|y\| \geq 1-\delta$, then $T_{2} \in L(1)$. When $\|x\|<1-\delta$ and $\|y\|<1-\delta$, we get

$$
\begin{aligned}
\left\|T_{2} x-T_{2} y\right\|^{2} & =\int_{0}^{1}(x(t)-y(t)+\|y\|-\|x\|)^{2} d t \\
& \leq 2 \int_{0}^{1}(x(t)-y(t))^{2} d t+2 \int_{0}^{1}(\|y\|-\|x\|)^{2} d t \\
& \leq 4\|x-y\|^{2} .
\end{aligned}
$$

Hence

$$
\left\|T_{2} x-T_{2} y\right\| \leq 2\|x-y\| .
$$

Let us estimate $\inf _{x \in B^{+}}\left\|T_{2} x\right\|$. Observe that if $\|x\| \geq 1-\delta$, then

$$
\left\|T_{2} x\right\|=\|x\| \geq 1-\delta
$$

If $\|x\|<1-\delta$, then

$$
\begin{aligned}
\left\|T_{2} x\right\|^{2} & =\int_{0}^{1}(1-\delta-\|x\|+x(t))^{2} d t \\
& =(1-\delta-\|x\|)^{2}+2(1-\delta-\|x\|) \int_{0}^{1} x(t) d t+\int_{0}^{1}(x(t))^{2} d t \\
& \geq 2\|x\|^{2}-2(1-\delta)\|x\|+(1-\delta)^{2} .
\end{aligned}
$$

The above minimum is attained for $\|x\|=\frac{1-\delta}{2}$ and is equal to $\frac{(1-\delta)^{2}}{2}$, which finally shows that

$$
\inf _{x \in B^{+}}\left\|T_{2} x\right\| \geq \frac{1-\delta}{\sqrt{2}} .
$$

Let $C=B^{+}(1) \backslash B^{+}\left(\frac{1-\delta}{\sqrt{2}}\right)$. Suppose that a mapping $T_{3}: \bar{C} \rightarrow S^{+}$is the radial projection, i.e.,

$$
\left(T_{3} x\right)(t)=\frac{x(t)}{\|x\|}
$$


From the properties of the radial projection we get that $T_{3} \in L\left(\frac{\sqrt{2}}{1-\delta}\right)$. We define the above-mentioned mapping $\widetilde{T}: B \rightarrow S^{+}$as the composition

$$
\widetilde{T}=T \circ T_{3} \circ T_{2} \circ T_{1},
$$

where $T$ is the mapping from the previous lemma. It is easy to see that

$$
\widetilde{T} \in L\left(\frac{2 \sqrt{2} \alpha^{\frac{3}{2}}}{1-\delta}\right) \quad \text { for } \alpha \geq 2,
$$

or equivalently, after simple calculations,

$$
\widetilde{T} \in L\left(\sqrt{2}(\alpha+1) \alpha^{\frac{3}{2}}\right) \quad \text { for } \alpha \geq 2 .
$$

The minimal displacement of a map $\widetilde{T}$ can be evaluated as follows:

$$
d_{\widetilde{T}}=\inf _{x \in B}\|x-\widetilde{T} x\| \geq \inf _{x \in B^{+}(1)}\|x-\widetilde{T} x\|=\inf _{x \in B^{+}(1)}\left\|x-T \circ T_{3} \circ T_{2} x\right\| .
$$

Observe that if $x \in B^{+}(1)$ and $\|x\|<1-\delta$, then

$$
\|x-\widetilde{T} x\|>\delta .
$$

If $x \in B^{+}(1)$ and $\|x\| \geq 1-\delta$, then

$$
\begin{aligned}
\|x-\widetilde{T} x\| & =\left\|x-T \circ T_{3} \circ T_{2} x\right\|=\left\|x-T \circ T_{3} x\right\|=\left\|x-T\left(\frac{x}{\|x\|}\right)\right\| \\
& \geq\left\|\frac{x}{\|x\|}-T\left(\frac{x}{\|x\|}\right)\right\| \sqrt{1-\left(\frac{\left\|\frac{x}{\|x\|}-T\left(\frac{x}{\|x\|}\right)\right\|}{2}\right)^{2}} \\
& =d_{T} \sqrt{1-\left(\frac{d_{T}}{2}\right)^{2}}=\delta=\frac{\alpha-1}{\alpha+1},
\end{aligned}
$$

which finally shows that

$$
\psi_{L^{2}(0,1)}\left(\sqrt{2}(\alpha+1) \alpha^{\frac{3}{2}}\right) \geq \frac{\alpha-1}{\alpha+1} \quad \text { for } \alpha \geq 2 .
$$

Observe that if $\alpha \rightarrow \infty$, then $\frac{\alpha-1}{\alpha+1} \rightarrow 1$, which shows that the above estimate is good for large $\alpha$.

\section{RETRACTION}

In this section we give the first completely constructive example of a Lipschitz retraction in the space $H$. Let $P: X \rightarrow B$ be the radial projection, i.e.,

$$
P x= \begin{cases}x & \text { if } x \in B, \\ \frac{x}{\|x\|} & \text { if } x \notin B .\end{cases}
$$

Denoting by $P(X)$ the Lipschitz constant of such a projection, it is known that $P(H)=1$ and $1 \leq P(X) \leq 2$ for any space $X$ [16]. We start with the following technical lemma, which holds in all infinite-dimensional Banach spaces.

Lemma 3.4. Suppose that there exists a homotopy $G:[0,1] \times S \rightarrow S$ such that for any $x \in S$, the following conditions hold:

$$
G(0, x)=x \quad \text { and } \quad G(1, x) \equiv x_{0} \in S .
$$

Assume that the homotopy $G$ is Lipschitz with constants $M$ and $N$, i.e., for every $x, y \in S$ and $c, d \in[0,1]$, the following condition holds:

$$
\|G(c, x)-G(d, y)\| \leq M|c-d|+N\|x-y\| .
$$


Then there exists a retraction $R: B \rightarrow S$ of a class $R \in L\left(\frac{P(X) N}{r}\right)$, where $r \in(0,1)$ is a solution of the equation

$$
\frac{P(X) N}{r}=\frac{M-P(X) N \ln r}{1-r} .
$$

Proof. Define a retraction $R: B \rightarrow S$ for $r \in(0,1)$ as follows:

$$
R x= \begin{cases}x_{0} & \text { if }\|x\| \leq r, \\ G\left(1-f(\|x\|), \frac{x}{\|x\|}\right) & \text { if }\|x\|>r,\end{cases}
$$

where $f$ is any increasing, convex and differentiable function on the interwal $[r, 1]$ such that $f(r)=0, f(1)=1$. Observe that the retraction $R$ is Lipschitz. For any $x, y \in B$ such that $\|x\| \geq r,\|y\| \geq r$ we have

$$
\begin{aligned}
\|R x-R y\| & =\left\|G\left(1-f(\|x\|), \frac{x}{\|x\|}\right)-G\left(1-f(\|y\|), \frac{y}{\|y\|}\right)\right\| \\
& \leq M|f(\|x\|)-f(\|y\|)|+N\left\|\frac{x}{\|x\|}-\frac{y}{\|y\|}\right\| \\
& \leq M f^{\prime}(\max \{\|x\|,\|y\|\})|\|x\|-\|y\||+\frac{P(X) N}{\max \{\|x\|,\|y\|\}}\|x-y\| \\
& \leq \max _{s \in[r, 1]}\left\{M f^{\prime}(s)+\frac{P(X) N}{s}\right\}\|x-y\| .
\end{aligned}
$$

To optimize the Lipschitz constant of a retraction $R$, observe that

$$
\int_{r}^{1}\left(M f^{\prime}(t)+\frac{P(X) N}{t}\right) d t=M-P(X) N \ln r
$$

does not depend on the choice of a function $f$. The Lipschitz constant will be minimal if the function satisfies a condition

$$
M f^{\prime}(t)+\frac{P(X) N}{t}=\frac{M-P(X) N \ln r}{1-r} .
$$

We get

$$
\|R x-R y\| \leq \frac{P(X) N}{r}\|x-y\|,
$$

where $r$ is a solution of the equation

$$
\frac{P(X) N}{r}=\frac{M-P(X) N \ln r}{1-r} .
$$

Observe that such a solution exists and is unique because the above functions are continuous on the interval $(0,1)$ and one is strictly increasing while the other is strictly decreasing.

Now we can proceed to the construction of a Lipschitz retraction in the Hilbert space $L^{2}(0,1)$.

Construction 3.5. Define a homotopy $G:[0,1] \times S \rightarrow S$ as follows:

$$
G(c, x)=\frac{(1-c) x+c T \circ T_{1} x}{\left\|(1-c) x+c T \circ T_{1} x\right\|},
$$

where the map $T$ is the map from Lemma 2.2 and the map $T_{1}$ is from the proof of Theorem 2.3. We show that the homotopy $G$ is well defined and

$$
\inf _{c \in[0,1]} \inf _{x \in S}\left\|(1-c) x+c T \circ T_{1} x\right\|>0 .
$$


Observe that

$$
\inf _{c \in[0,1]} \inf _{x \in S}\left\|(1-c) x+c T \circ T_{1} x\right\|=\inf _{c \in[0,1]} \inf _{x \in S^{+}}\|(1-c) x-c T x\|
$$

and

$$
\begin{aligned}
d_{T}^{2} & =\inf _{x \in S^{+}}\|x-T x\|^{2} \\
& \leq\|x-T x\|^{2} \\
& =2\|x\|^{2}-2\langle x, T x\rangle+\|T x\|^{2} \\
& =2-2\langle x, T x\rangle .
\end{aligned}
$$

Using this estimate we get

$$
\begin{aligned}
\|(1-c) x-c T x\|^{2} & =(1-c)^{2}\|x\|^{2}-2 c(1-c)\langle x, T x\rangle+c^{2}\|T x\|^{2} \\
& =1-c(1-c)\left(4-d_{T}^{2}\right) \\
& \geq \frac{d_{T}^{2}}{4}>0
\end{aligned}
$$

The homotopy $G$ is Lipschitz. Indeed,

$$
\begin{aligned}
\|G(c, x)-G(d, y)\| \leq & \|G(c, x)-G(d, x)\|+\|G(d, x)-G(d, y)\| \\
\leq & \frac{2|c-d|}{\inf _{c \in[0,1]} \inf _{x \in S}\left\|(1-c) x+c T \circ T_{1} x\right\|} \\
& +\sup _{c \in[0,1]} \frac{1+c(k-1)}{\inf _{z \in S^{+}}\|(1-c) z-c T z\|}\|x-y\|,
\end{aligned}
$$

where $k=\alpha^{\frac{3}{2}}$. Since for $c \in[0,1]$ we have

$$
\|(1-c) x-c T x\|=\|c x-(1-c) T x\|,
$$

we obtain

$$
\begin{aligned}
\|H(c, x)-H(d, y)\| & \leq \frac{4}{d_{T}}|c-d|+\sup _{c \in\left[\frac{1}{2}, 1\right]} \frac{1+c(k-1)}{\inf _{z \in S^{+}}\|(1-c) z-c T z\|}\|x-y\| \\
& =M(\alpha)|c-d|+N(\alpha)\|x-y\|,
\end{aligned}
$$

where (after calculations)

$$
M(\alpha)=\frac{2 \sqrt{2(\alpha+1)}}{\sqrt{\alpha}-1} \quad \text { and } \quad N(\alpha)=\sup _{c \in\left[\frac{1}{2}, 1\right]} \frac{1+c\left(\alpha^{\frac{3}{2}}-1\right)}{\sqrt{1-2 c(1-c) \frac{(\sqrt{\alpha}+1)^{2}}{\alpha+1}}} .
$$

Now define a mapping $T_{2}: B^{+}(r) \rightarrow B^{+}(r)$ by

$$
\left(T_{2} x\right)(t)=r-\|x\|+x(t) .
$$

From the proof of Theorem 2 we conclude that

$$
T_{2} \in L(2) \quad \text { and } \quad \inf _{x \in B^{+}(r)}\left\|T_{2} x\right\| \geq \frac{r}{\sqrt{2}} .
$$

Now we can define a retraction $R: B \rightarrow S$ by

$$
(R x)(t)= \begin{cases}\left(T \circ\left(\frac{T_{2}}{\left\|T_{2}\right\|}\right) \circ T_{1} x\right)(t) & \text { if }\|x\| \leq r, \\ G\left(1-f(\|x\|), \frac{x(t)}{\|x\|}\right) & \text { if }\|x\|>r,\end{cases}
$$

where $f$ is any increasing, convex and differentiable function defined on the interval $[r, 1]$ such that $f(r)=0$ and $f(1)=1$. 
Observe that if $\|x\| \leq r$ and $\|y\| \leq r$ we get

$$
\begin{aligned}
\|R x-R y\| & \leq\left\|T \circ\left(\frac{T_{2}}{\left\|T_{2}\right\|}\right) \circ T_{1} x-T \circ\left(\frac{T_{2}}{\left\|T_{2}\right\|}\right) \circ T_{1} y\right\| \\
& \leq \frac{2 \sqrt{2} k}{r}\|x-y\|=2 \sqrt{2} \frac{\alpha^{\frac{3}{2}}}{r}\|x-y\| .
\end{aligned}
$$

If $\|x\|>r$ and $\|y\|>r$, using the previous lemma we have

$$
\|R x-R y\| \leq \frac{N(\alpha)}{r}\|x-y\|,
$$

where $r$ is a solution of the equation

$$
\frac{N(\alpha)}{r}=\frac{M(\alpha)-N(\alpha) \ln r}{1-r} .
$$

Numerical experiments show that for $\alpha=2.535$ we get a retraction with the minimal Lipschitz constant slightly less than 32.26 . This implies the following.

Corollary 3.6. In the infinite-dimensional Hilbert space $H$,

$$
k_{0}(H)<32.26 \text {. }
$$

The problem of exact evaluation of $k_{0}(X)$ for at least one space is still open.

\section{REFERENCES}

[1] Y. Benyamini and Y. Sternfeld, Spheres in infinite-dimensional normed spaces are Lipschitz contractible, Proc. Amer. Math. Soc. 88 (1983), 439-445. MR 85h:46028

[2] K. Bolibok, Constructions of Lipschitzian mappings with non-zero minimal displacement in spaces $L^{1}(0,1)$ and $L^{2}(0,1)$, Ann. Univ. Marie Curie-Sklodowska Sect. A 50 (1996), 25-31. MR 98j: 47123

[3] K. Bolibok, Construction of a Lipschitz retraction in the space $c_{0}$, Ann. Univ. Marie CurieSklodowska Sect. A. 51 (1997), 43-46. MR 99m:46029

[4] K. Bolibok and K. Goebel, A note on minimal displacement and retraction problems, J. Math. Anal. Appl. 206 (1997), 308-314. MR 97m:47078

[5] C. Franchetti, Lipschitz maps and the geometry of the unit ball in normed spaces, Arch. Math. 46 (1986), 76-84. MR 87g:46025

[6] M. Furi and M. Martelli, On the minimal displacement of points under alpha-Lipschitz maps in normed spaces, Boll. Un. Mat. Ital. 9 (1974), 791-799. MR 51:6509

[7] K. Goebel, On the minimal displacement of points under Lipschitz mappings, Pacific J. Math. 45 (1973), 151-163. MR 48:7050

[8] K. Goebel, Metric environment of the topological fixed point theorems, "Handbook of Metric Fixed Point Theory", Kluwer, Dordrecht, 2001, 577-611. MR 2003e:47096

[9] K. Goebel and W. A. Kirk, Topics in Metric Fixed Point Theory, Cambridge University Press, Cambridge, 1990. MR 92c:47070

[10] K. Goebel and T. Komorowski, Retracting balls onto spheres and minimal displacement problems, Fixed Point Theory and Applications, Pitman Research Notes in Math., Longman Sci. Tech., Harlow, 1991, 155-172. MR 93b:47110

[11] T. Komorowski and J. Wośko, A remark on the retracting of a ball onto a sphere in an infinite-dimensional Hilbert space, Math. Scand. 67 (1990), 223-226. MR 92e:58012

[12] P. K. Lin and Y. Sternfeld, Convex sets with the Lipschitz fixed point property are compact, Proc. Amer. Math. Soc. 93 (1985), 633-639. MR 86c:47074

[13] B. Nowak, On the Lipschitz retraction of the unit ball in infinite-dimensional Banach spaces onto its boundary, Bull. Acad. Polon. Sci. Sér. Sci. Math. 27 (1979), 861-864. MR 82g:58008

[14] S. Reich, Minimal displacement of points under weakly inward pseudo-Lipschitzian mappings I, Atti. Accad. Naz. Linzei Rend. Cl. Sci. Fis. Mat. Natur. 59 (1975), 40-44. MR 56:9345 
[15] S. Reich, Minimal displacement of points under weakly inward pseudo-Lipschitzian mappings II, Atti. Accad. Naz. Linzei Rend. Cl. Sci. Fis. Mat. Natur. 60 (1976), 95-96. MR 58:7264

[16] R. L. Thele, Some results on the radial projection in Banach spaces, Proc. Amer. Math. Soc. 42 (1974), 483-486. MR 48:6892

Institute of Mathematics, Maria Curie - SkŁodowska University, 20-031 Lublin, POLAND

E-mail address: bolibok@golem.umcs.lublin.pl 